\title{
Dynamical Behaviors of the Tumor-immune System in a Stochastic Environment
}

\author{
Xiaoyue Li* Guoting Song ${ }^{\dagger} \quad$ Yang Xia $^{\ddagger} \quad$ Chenggui Yuan $^{\S}$
}

\begin{abstract}
This paper investigates dynamical behaviors of the tumor-immune system perturbed by environmental noise. The model describes the response of the cytotoxic $\mathrm{T}$ lymphocyte to the growth of an immunogenic tumour. The main methods are stochastic Lyapunov analysis, comparison theorem for stochastic differential equations (SDEs) and strong ergodicity theorem. Firstly, we prove the existence and uniqueness of the global positive solution for the tumor-immune system. Then we go a further step to study the boundaries of moments for tumor cells and effector cells and the asymptotic behavior in the boundary equilibrium points. Furthermore, we discuss the existence and uniqueness of stationary distribution and stochastic permanence of the tumor-immune system. Finally, we give several examples and numerical simulations to verify our results.
\end{abstract}

AMS Subject Classification: $60 \mathrm{H} 10 \cdot 65 \mathrm{C} 20$

Keywords: Tumor-immune system.Stochastic permanence-Comparison theorem · Invariant measure.Ergodicity

\section{Introduction}

At present, cancer is considered to be one of the most complicated diseases to be treated clinically and one of the most dreadful killers in the world today. Keeping in mind its devastating nature, a great deal of human and economic resources are devoted to the research on cancer biology and subsequent development of proper therapeutic measures. Surgery,

${ }^{*}$ School of Mathematics and Statistics, Northeast Normal University, Changchun, Jilin, 130024, China. Research of this author was supported by National Natural Science Foundation of China (11171056, 11471071, 11671072), the Natural Science Foundation of Jilin Province (No.20170101044JC), the Education Department of Jilin Province (No.JJKH20170904KJ), the Fundamental Research Funds for the Central Universities.

${ }^{\dagger}$ School of Mathematics and Statistics, Northeast Normal University, Changchun, Jilin, 130024, China.

${ }^{\ddagger}$ School of Mathematics and Statistics, Northeast Normal University, Changchun, Jilin, 130024, China; Shenzhen Foreign Languages School, Shenzhen, Guangdong, 518000, China

${ }^{\S}$ Department of Mathematics, Swansea University, Bay Campus, SA1 8EN, UK. 
radiation therapy, and chemotherapy are the three traditional therapy procedures that are practised for treatment of cancer. However, all these procedures are characterized by a relatively low efficacy and high toxicity for the patient. Therefore, compared with traditional treatment methods, emerging immunotherapy has great development prospects. Immunotherapy, also known as biological therapy, usually refers to the use of cytokines, a protein hormone that mediate both natural and specific immunity to induce antitumor responses of immune system.

Mathematical models of tumour-immune system and their dynamical behaviors [1, 3], help us to understand better how host immune cells and cancerous cells evolve and interact. In order to get closer to reality more and more tumour-immune models have been studied, for instance, $[6,8,10,21,24,31,35,36,37]$ and reference therein. It's worth noticing that a classical mathematical simplified tumour-immune model

$$
\left\{\begin{aligned}
\mathrm{d} x(t) & =\left(\sigma+\frac{\rho x(t) y(t)}{\eta+y(t)}-\mu x(t) y(t)-\delta x(t)\right) \mathrm{d} t, \\
\mathrm{~d} y(t) & =\left(\alpha y(t)-\beta y^{2}(t)-x(t) y(t)\right) \mathrm{d} t
\end{aligned}\right.
$$

is proposed to simulate the interaction of the cytotoxic $\mathrm{T}$ lymphocyte with immunogenic tumor cells and took into account the inactivation of effector cells as well as the penetration of effector cells into tumor cells by [22], where $x$ represents non-dimensional local concentration of effector cells (EC), $y$ represents the non-dimensional local concentration of tumor cells (TC). Local and global bifurcations for the parameters $\delta$, the effector cell "death rate", and $\sigma$, the baseline effector cell "source rate", were calculated, and the possible connection between two different mechanisms of the tumor: tumor dormancy and sneaking through is illustrated. Yafia [37] studied the stability of the equilibriums and proved the existence of a family of periodic solutions bifurcating from the nontrivial steady state of the Kuznetsov-Taylor model with a delay. More complete bibliography about the evolution of cells and the relevant role of cellular phenomena in directing the body toward recovery or toward illness can be found in [7, 29, 32].

In the tumor tissue, the growth rate and cytotoxic parameters are influenced by many environmental factors, e.g. the degree of vascularization of tissues, the supply of oxygen, the supply of nutrients, the immunological state of the host, chemical agents, temperature, radiations, gene expression, protein synthesis and antigen shedding from the cell surface, etc. Due to the complexity, it is unavoidable that in the course of time the parameters of the system undergo random variations which give them a stochastic character $[12,14$, $15,25]$. Inclusion of randomness in mathematical models of biological and biochemical processes is thus necessary for better understanding of mechanisms which govern the biological systems. Considering the impact of the stochastic volatility of environment, we assume that environmental fluctuations mainly affect the culling rate of effector cells $\delta$ and the intrinsic growth rate of tumor cells $\alpha$

$$
-\delta \mathrm{d} t \rightarrow-\delta \mathrm{d} t+\sigma_{1} \mathrm{~d} B_{1}(t), \quad \alpha \mathrm{d} t \rightarrow \alpha \mathrm{d} t+\sigma_{2} \mathrm{~d} B_{2}(t),
$$

where $B_{1}(t)$ and $B_{2}(t)$ are the 1-dimensional Brown motion and independent, and $\sigma_{1}$ and $\sigma_{2}$ denote the intensity of white noises. Thus the stochastic tumor-immune model is 
described by the following SDE

$$
\left\{\begin{array}{l}
\mathrm{d} x(t)=\left(\sigma+\frac{\rho x(t) y(t)}{\eta+y(t)}-\mu x(t) y(t)-\delta x(t)\right) d t+\sigma_{1} x(t) \mathrm{d} B_{1}(t), \\
\mathrm{d} y(t)=\left(\alpha y(t)-\beta y^{2}(t)-x(t) y(t)\right) d t+\sigma_{2} y(t) \mathrm{d} B_{2}(t),
\end{array}\right.
$$

with an initial value $x(0)=x_{0}>0, y(0)=y_{0}>0$. Based on the actual background of the model, we assume that $\sigma_{1}, \sigma_{2}$ and all other parameters are non-negative real numbers. Obviously, the model (1.2) degenerates into (1.1) if $\sigma_{1}=0, \sigma_{2}=0$.

In the last years, stochastic growth models for cancer cells have been developed, one can see $[2,13,34]$ and reference therein. Lyapunov exponent method and Fokker-Planck method are used to investigate the stability of the stochastic models by numerical simulations. Mukhopadhyay and Bhattacharyya [28] analyzed the stochastic stability for a stochastic virus-tumor-immune model. Oana, Dumitru and Riccardo [30] studied the stochastic stability of the stochastic Kuznetsov-Taylor model near their equilibriums. Li and Cheng [23] established the scalar tumor growth model describing the interaction and competition between the tumor cells based on the Michaelis-Menten enzyme kinetics, and gave the threshold conditions for extinction, weak persistence and stochastic persistence of tumor cells by the rigorous theoretical proofs, to name a few.

In this paper our main aim is to investigate the stochastic Kuznetsov-Taylor tumorimmune model (1.2), which describes the response of the cytotoxic $\mathrm{T}$ lymphocyte to the growth of immunogenic tumor cells. Combing the stochastic Lyapunov analysis with the comparison principle for SDEs and making use of the strong ergodicity theorem, we discuss the asymptotic behaviors including the stochastic ultimately boundedness in moment, the limit distribution as well as the ergodicity. Especially, it is pointed out that when tumor cells subject to strong stochastic perturbations, the density of tumor cells is exponentially decreasing while the density of effector cells tends to the stationary distribution. On the other hand, under weak environmental noises of the tumor cells, the existence and uniqueness of the stationary distribution with the support set $\mathbb{R}_{+}^{2}$ is yielded, which implies that tumor cells and immune cells are stochastically permanence and approach a dormant steady state. These obtained judgement criteria on extinction and permanence will provide us some inspirations on how to make more effective and precise therapeutic schedule to eliminate tumor cells and improve the treatment of cancer.

Compared with the results on the stochastic Kuznetsov-Taylor model [30] and the scalar tumor growth model [23], our model not only considers the stimulated accumulation of effector cells due to the presence of the tumor but also predicts the stochastic permanence and the ergodicity by the rigorous theoretical proofs. Although those of the deterministic model (1.1) are rich in [22], the dynamical behaviors of the stochastic model (1.2) are different completely, especially, as the intensity of the tumor noises is large, see Examples 5.1 and 5.2. The fact is revealed that the stochastic environmental perturbation to the tumor cells cannot be ignored, which is the key factor to eliminate the tumor cells.

The rest of the paper is arranged as follow. Section 2 gives some notations and proves the existence of the unique global positive solution. Section 3 obtains the ultimate 
moment boundedness of the global positive solution. Section 4 yields the ergodicity of tumor cells and effector cells in the stochastic tumor-immune model which implies the stochastic permanence of cells. Section 5 presents a couple of examples and numerical simulations to illustrate our results. Section 6 concludes this paper.

\section{Global positive solution}

Throughout this paper, let $\left(\Omega, \mathcal{F},\left\{\mathcal{F}_{t}\right\}_{t \geq 0}, \mathbb{P}\right)$ be a complete filtered probability space with $\left\{\mathcal{F}_{t}\right\}_{t \geq 0}$ satisfying the usual conditions (that is, it is right continuous and $\mathcal{F}_{0}$ contains all $\mathbb{P}$ null sets). Let $B(t)=\left(B_{1}(t), B_{2}(t)\right)^{T}$ be a 2-dimensional Brownian motion defined on the probability space. Let $|\cdot|$ denote the Euclidean norm in $\mathbb{R}^{2}$. Also let $\mathbb{R}_{+}=\{x \in \mathbb{R} \mid x>0\}$ and $\mathbb{R}_{+}^{2}=\left\{(x, y) \in \mathbb{R}^{2} \mid x>0, y>0\right\}$. Let $C$ denote a generic positive constant whose value may change in different appearances. Moreover, let $C^{2,1}\left(\mathbb{R}^{2} \times \mathbb{R}_{+} ; \mathbb{R}_{+}\right)$denote the family of all nonnegative functions $V(x, t)$ on $\mathbb{R}^{2} \times \mathbb{R}_{+}$which are continuously twice differentiable in $x$ and once differentiable in $t$. For each $V \in C^{2,1}\left(\mathbb{R}^{2} \times \mathbb{R}_{+} ; \mathbb{R}_{+}\right)$, define an operator $\mathcal{L}$ such that $\mathcal{L} V: \mathbb{R}^{2} \times \mathbb{R}_{+} \rightarrow \mathbb{R}$ with the form

$$
\begin{aligned}
\mathcal{L} V(x, y, t)= & V_{t}(x, y, t)+V_{x}(x, y, t)\left(\sigma+\frac{\rho x y}{\eta+y}-\mu x y-\delta x\right) \\
& +V_{y}(x, y, t)\left(\alpha y-\beta y^{2}-x y\right) \\
& +\frac{1}{2}\left(V_{x x}(x, y, t) \sigma_{1}^{2} x^{2}+V_{y y}(x, y, t) \sigma_{2}^{2} y^{2}\right) .
\end{aligned}
$$

Since $x(t)$ represents the density of EC, $y(t)$ represents the density of TC, both $x(t)$ and $y(t)$ in (1.2) should be positive. The theorem below gives an affirmative answer.

Theorem 2.1 For any initial value $\left(x_{0}, y_{0}\right) \in \mathbb{R}_{+}^{2}$, the equation (1.2) has a unique global positive solution $(x(t), y(t))$ for all $t \geq 0$ with probability one.

Proof. Due to the local Lipschitz continuity of the coefficients, by [26, p.91, Theorem 3.3.15], there is a unique local solution $(x(t), y(t))\left(t \in\left[0, \tau_{e}\right)\right)$ with any given initial value $\left(x_{0}, y_{0}\right) \in \mathbb{R}_{+}^{2}$, where $\tau_{e}$ is the explosion time, namely, it is a stopping time such that $0 \leq \tau_{e} \leq \infty$ a.s. and $\limsup _{t \rightarrow \tau_{e}}\left(x^{2}(t)+y^{2}(t)\right)=\infty$ whenever $\tau_{e}<\infty$ [26, p.90, Definition 3.3.14]. Choose an $m_{0} \geq 1$ such that $x_{0} \in\left(1 / m_{0}, m_{0}\right), y_{0} \in\left(1 / m_{0}, m_{0}\right)$. For any positive $m \geq m_{0}$, define the stopping time as follows

$$
\tau_{m}=\inf \left\{t \in\left[0, \tau_{e}\right): \min \{x(t), y(t)\} \leq \frac{1}{m} \text { or } \max \{x(t), y(t)\} \geq m\right\}
$$

We set $\inf \emptyset=\infty$, clearly, $\tau_{m} \leq \tau_{e}$, and $\tau_{m}$ is increasing as $m \rightarrow \infty$. Let $\tau_{\infty}=\lim _{m \rightarrow \infty} \tau_{m}$, $\tau_{\infty} \leq \tau_{e}$ a.s. If we can prove that $\tau_{\infty}=\infty$ a.s. then $\tau_{e}=\infty$ a.s. This implies that the solution is not only positive but also global.

Here we give a proof by contradiction. Suppose that $\tau_{\infty}=\infty$ a.s. doesn't hold, then there exist constants $T>0$ and $\varepsilon \in(0,1)$ such that

$$
\mathbb{P}\left(\tau_{\infty} \leq T\right)>\varepsilon
$$


This implies that exists an $m_{1} \geq m_{0}$ such that for all $m \geq m_{1}$

$$
\mathbb{P}\left(\tau_{m} \leq T\right) \geq \frac{\varepsilon}{2}
$$

Define

$$
V(x, y)=(x+1-\log x)+(y+1-\log y), \quad \forall(x, y) \in \mathbb{R}_{+}^{2} .
$$

Using the Itô formula, we have

$$
\mathbb{E}\left[V\left(x\left(\tau_{m} \wedge T\right), y\left(\tau_{m} \wedge T\right)\right)\right]=V\left(x_{0}, y_{0}\right)+\mathbb{E} \int_{0}^{\tau_{m} \wedge T} \mathcal{L} V(x(t), y(t)) \mathrm{d} t,
$$

where

$$
\begin{aligned}
\mathcal{L} V(x, y)= & \left(\sigma+\delta-\alpha+\frac{1}{2} \sigma_{1}^{2}+\frac{1}{2} \sigma_{2}^{2}\right)+\frac{\rho x y}{\eta+y}+x+(\mu+\alpha+\beta) y \\
& -\mu x y-\delta x-\frac{\sigma}{x}-\frac{\rho y}{\eta+y}-\beta y^{2}-x y \\
\leq & \left(\sigma+\delta+\frac{1}{2} \sigma_{1}^{2}+\frac{1}{2} \sigma_{2}^{2}\right)+(\rho+1) x+(\mu+\alpha+\beta) y . \\
\leq & v_{1}+2(\rho+1)(x+1-\log x)+2(\mu+\alpha+\beta)(y+1-\log y) \\
\leq & v_{1}+v_{2} V(x, y)
\end{aligned}
$$

with $v_{1}=\sigma+\delta+\frac{1}{2} \sigma_{1}^{2}+\frac{1}{2} \sigma_{2}^{2}, \quad v_{2}=2(\rho+1+\mu+\alpha+\beta)$. This, together with (2.4), implies

$$
\begin{aligned}
\mathbb{E}\left[V\left(x\left(\tau_{m} \wedge T\right), y\left(\tau_{m} \wedge T\right)\right)\right] & \leq V\left(x_{0}, y_{0}\right)+v_{1} T+v_{2} \mathbb{E} \int_{0}^{\tau_{m} \wedge T} V(x(t), y(t)) \mathrm{d} t \\
& =V\left(x_{0}, y_{0}\right)+v_{1} T+v_{2} \mathbb{E} \int_{0}^{T} \mathbf{I}_{\left.\left[0, \tau_{m}\right]\right]}(t) V(x(t), y(t)) \mathrm{d} t \\
& \leq V\left(x_{0}, y_{0}\right)+v_{1} T+v_{2} \mathbb{E} \int_{0}^{T} V\left(x\left(t \wedge \tau_{m}\right), y\left(t \wedge \tau_{m}\right)\right) \mathrm{d} t \\
& =V\left(x_{0}, y_{0}\right)+v_{1} T+v_{2} \int_{0}^{T} \mathbb{E} V\left(x\left(t \wedge \tau_{m}\right), y\left(t \wedge \tau_{m}\right)\right) \mathrm{d} t,
\end{aligned}
$$

where $\mathbf{I}_{A}(\cdot)$ is the indicate function of a set $A$. The Gronwall inequality yields that

$$
\mathbb{E}\left[V\left(x\left(\tau_{m} \wedge T\right), y\left(\tau_{m} \wedge T\right)\right)\right] \leq\left(V\left(x_{0}, y_{0}\right)+v_{1} T\right) e^{v_{2} T} .
$$

Let $\Omega_{m}=\left\{\omega: \tau_{m} \leq T\right\}$, then $\forall \omega \in \Omega_{m}$, at least one of $x\left(\tau_{m}(\omega) \wedge T\right)$ and $y\left(\tau_{m}(\omega) \wedge T\right)$ is equal to $1 / m$ or $m$. Hence, we have

$$
(m+1-\log m) \wedge\left(\frac{1}{m}+1+\log m\right) \leq V\left(x\left(\tau_{m} \wedge T\right), y\left(\tau_{m} \wedge T\right)\right) .
$$

Due to (2.3) and (2.5), we arrive at

$$
\begin{aligned}
\frac{\varepsilon}{2}(m+1-\log m) \wedge\left(\frac{1}{m}+1+\log m\right) & \leq \mathbb{E}\left[\mathbf{I}_{\Omega_{m}}(\omega) V\left(x\left(\tau_{m} \wedge T\right), y\left(\tau_{m} \wedge T\right)\right)\right] \\
& \leq\left(V\left(x_{0}, y_{0}\right)+v_{1} T\right) e^{v_{2} T}
\end{aligned}
$$


On the other hand, one observes

$$
\lim _{m \rightarrow \infty}(m+1-\log m) \wedge\left(\frac{1}{m}+1+\log m\right)=\infty .
$$

Taking $m \rightarrow \infty$ in (2.6), we obtain

$$
\infty \leq\left(V\left(x_{0}, y_{0}\right)+v_{1} T\right) e^{v_{2} T}<\infty,
$$

which results in a contradiction. The proof is therefore complete.

\section{Moment boundedness}

Based on the existence result of positive solutions, this section focuses on the asymptotic estimation of the moments of $x(t)$ and $y(t)$. We borrow the idea of looking at the boundary to analyze the asymptotic behaviors $[9,11,16,17]$. The method of looking at the boundary has been shown to be very effective since it enables ones to get not only sufficient but close to necessary conditions $[9,11]$. Its generalization to high dimensional Kolmogorov systems are referred to $[16,17]$. In order to discuss the asymptotic upper boundary of $\mathbb{E}\left[y^{k}(t)\right]$, we look at the growth rule of $y(t)$ on the boundary $x(t)=0$. So we introduce an auxiliary process $\psi(t)$ described by

$$
\left\{\begin{array}{l}
\mathrm{d} \psi(t)=\psi(t)[\alpha-\beta \psi(t)] \mathrm{d} t+\sigma_{2} \psi(t) \mathrm{d} B_{2}(t) \\
\psi(0)=y_{0}>0
\end{array}\right.
$$

where $B_{2}(t)$ is the Brownian motion defined in (1.2). By utilizing a comparison theorem, one observes that $0<y(t) \leq \psi(t)$ for all $t \geq 0$ a.s. The following result is taken from [5], we cite it as a lemma.

Lemma 3.1 [5, Lemma 2.1, p.729] Let $\psi(t)$ be the solution of (3.1). Then it holds that for any $k>1$,

$$
\mathbb{E} \psi^{k}(t) \leq\left[\frac{1}{y_{0}} e^{-\left(\alpha+\frac{k-1}{2} \sigma_{2}^{2}\right) t}+\frac{2 \beta}{2 \alpha+(k-1) \sigma_{2}^{2}}\left(1-e^{-\left(\alpha+\frac{k-1}{2} \sigma_{2}^{2}\right) t}\right)\right]^{-k} .
$$

Therefore, we have

$$
\limsup _{t \rightarrow \infty} \mathbb{E} \psi^{k}(t) \leq \varrho_{k}:=\left(\frac{2 \alpha+(k-1) \sigma_{2}^{2}}{2 \beta}\right)^{k}, \forall k>1 .
$$

We now investigate the asymptotic properties of the moments of $y(t)$.

Theorem 3.2 For any $k>1$, we have

$$
\limsup _{t \rightarrow+\infty} \mathbb{E}\left[y^{k}(t)\right] \leq \varrho_{k}
$$

For any $0<k \leq 1$, we have

$$
\limsup _{t \rightarrow+\infty} \mathbb{E}\left[y^{k}(t)\right] \leq\left(\varrho_{2}\right)^{\frac{k}{2}}
$$


Proof. Since $0<y(t) \leq \psi(t)$, for $k>1$, by Lemma 3.1, we have

$$
\limsup _{t \rightarrow+\infty} \mathbb{E}\left[y^{k}(t)\right] \leq \limsup _{t \rightarrow+\infty} \mathbb{E}\left[\psi^{k}(t)\right] \leq \varrho_{k} .
$$

Furthermore, for $0<k \leq 1$, by the Hölder inequality, we obtain

$$
\limsup _{t \rightarrow+\infty} \mathbb{E}\left[y^{k}(t)\right] \leq \limsup _{t \rightarrow+\infty}\left[\mathbb{E}\left[y^{2}(t)\right]\right]^{\frac{k}{2}} \leq\left(\varrho_{2}\right)^{\frac{k}{2}}
$$

as required.

Next, we continue to consider the asymptotic property of the moments of $x(t)$. By virtue of the interaction between $x(t)$ and $y(t)$ and the positivity of $y(t)$ we provide the following sufficient result for the moment boundedness of $x(t)$.

Theorem 3.3 For any $\theta \in\left(0,1+2 \delta / \sigma_{1}^{2}\right)$ and $c>[\rho / \eta-\mu]^{+}$,

$$
\limsup _{t \rightarrow \infty} \mathbb{E}\left[(1+x(t)+c y(t))^{\theta}\right] \leq L(c, \theta),
$$

where $L(c, \theta)$ is a positive constant dependent on $\theta$ and $c$, which is defined by (3.8) below.

Proof. Since the proof is rather technical we divide it into three steps.

Step 1. We estimate the upper bound of $\mathcal{L}\left[e^{\kappa t} V_{1}(x, y)\right]$. Define the function $f_{1}(y)=$ $\frac{\rho y}{\eta+y}-(\mu+c) y$ for any $y \geq 0$, then

$$
f_{1}^{\prime}(y)=\frac{-(\mu+c) y^{2}-2(\mu+c) \eta y+(\rho-\eta(\mu+c)) \eta}{(\eta+y)^{2}} .
$$

Solving $f_{1}^{\prime}(y)=0$, we obtain two roots

$$
y_{1}=-\eta-\sqrt{\frac{\eta \rho}{\mu+c}}<0, \quad y_{2}=-\eta+\sqrt{\frac{\eta \rho}{\mu+c}} .
$$

The condition $c>[\rho / \eta-\mu]^{+}$yields $y_{2}<0$. By virtue of $f_{1}^{\prime}(y)<0(\forall y>0)$ it follows that

$$
f_{1}(y)<f_{1}(0)=0, \quad \forall y>0 .
$$

For a fixed $\theta \in\left(0,1+2 \delta / \sigma_{1}^{2}\right)$, define

$$
V_{1}(x, y)=(1+x+c y)^{\theta}, \quad \forall(x, y) \in \mathbb{R}_{+}^{2} .
$$

By virtue of (2.1) and (3.3), computing $\mathcal{L} V_{1}(x, y)$ leads to

$$
\begin{aligned}
& \mathcal{L} V_{1}(x, y) \\
=\theta(1 & +x+c y)^{\theta-2}\left\{\left[\frac{\rho y}{\eta+y}-(\mu+c) y-\delta+\frac{\theta-1}{2} \sigma_{1}^{2}\right] x^{2}\right. \\
& +\left[-c(\beta+\mu+c) y^{2}+\left(c \alpha+\frac{c \rho y}{\eta+y}-c \delta-\mu-c\right) y+\frac{\rho y}{\eta+y}-\delta+\sigma\right] x \\
& \left.-c^{2} \beta y^{3}+\left(c^{2} \alpha-c \beta+\frac{\theta-1}{2} c^{2} \sigma_{2}^{2}\right) y^{2}+c(\alpha+\sigma) y+\sigma\right\} .
\end{aligned}
$$


The condition $0<\theta<1+2 \delta / \sigma_{1}^{2}$ implies $\delta+\frac{(1-\theta)}{2} \sigma_{1}^{2}>0$. Now, choose a positive constant $\kappa:=\kappa(\theta)$ sufficiently small such that

$$
L_{1}(\theta):=\delta+\frac{(1-\theta)}{2} \sigma_{1}^{2}-\frac{\kappa}{\theta}>0
$$

By the Itô formula,

$$
M_{v_{1}}(t):=e^{\kappa t} V_{1}(x(t), y(t))-V_{1}\left(x_{0}, y_{0}\right)-\int_{0}^{t} \mathcal{L}\left[e^{\kappa t} V_{1}(x(s), y(s))\right] \mathrm{d} s
$$

is a local martingale. By (3.4), we have

$$
\begin{aligned}
& \mathcal{L}\left[e^{\kappa t} V_{1}(x, y)\right] \\
= & \kappa e^{\kappa t} V_{1}(x, y)+e^{\kappa t} \mathcal{L} V_{1}(x, y) \\
\leq & \theta e^{\kappa t}(1+x+c y)^{\theta-2}\left\{\left[\frac{\rho y}{\eta+y}-(\mu+c) y-\left(\delta+\frac{1-\theta}{2} \sigma_{1}^{2}-\frac{\kappa}{\theta}\right)\right] x^{2}\right. \\
& +\left[-c(\beta+\mu+c) y^{2}+\left(c \alpha+c \rho-c \delta-\mu-c+\frac{2 c \kappa}{\theta}\right) y\right] x \\
& +\left(\rho-\delta+\sigma+\frac{2 \kappa}{\theta}\right) x-c^{2} \beta y^{3}+\left(c^{2} \alpha-c \beta+\frac{\theta-1}{2} c^{2} \sigma_{2}^{2}+\frac{c^{2} \kappa}{\theta}\right) y^{2} \\
& \left.+c\left(\alpha+\sigma+\frac{2 \kappa}{\theta}\right) y+\sigma+\frac{\kappa}{\theta}\right\} \\
\leq & \theta e^{\kappa t}(1+x+c y)^{\theta-2} W(x, y),
\end{aligned}
$$

where

$$
\begin{aligned}
W(x, y):= & -L_{1}(\theta) x^{2}+L_{2}(c, \theta) x-c^{2} \beta y^{3}+\left(c^{2} \alpha-c \beta+\frac{\theta-1}{2} c^{2} \sigma_{2}^{2}+\frac{c^{2} \kappa}{\theta}\right) y^{2} \\
& +c\left(\alpha+\sigma+\frac{2 \kappa}{\theta}\right) y+\sigma+\frac{\kappa}{\theta}, \\
L_{2}(c, \theta):= & \sup _{y \in \mathbb{R}_{+}}\left\{-c(\beta+\mu+c) y^{2}+\left(c \alpha+c \rho-c \delta-\mu-c+\frac{2 c \kappa}{\theta}\right) y+\rho-\delta+\alpha+\frac{2 \kappa}{\theta}\right\} .
\end{aligned}
$$

One observes that

$$
\lim _{x^{2}+y^{2} \rightarrow+\infty}(1+x+c y)^{\theta-2} W(x, y)=-\infty,
$$

which together with the continuity of $(1+x+c y)^{\theta-2} W(x, y)$ in $\mathbb{R}_{+}^{2}$ implies that

$$
L_{3}(c, \theta):=\theta \sup _{x, y \in \mathbb{R}_{+}}\left\{(1+x+c y)^{\theta-2} W(x, y)\right\}<+\infty .
$$

Thus, we have

$$
\mathcal{L}\left[e^{\kappa t} V_{1}(x, y)\right] \leq L_{3}(c, \theta) e^{\kappa t}
$$


Step 2. We show that

$$
\mathbb{E}\left[e^{\kappa t} V_{1}(x(t), y(t))\right]=\mathbb{E}\left[V_{1}\left(x_{0}, y_{0}\right)\right]+\mathbb{E} \int_{0}^{t} \mathcal{L}\left[e^{\kappa s} V_{1}(x(s), y(s))\right] \mathrm{d} s .
$$

In fact, let $n_{0}>0$ be sufficiently large for $x_{0}, y_{0}$ lying within the interval $\left(1 / n_{0}, n_{0}\right)$. For any constant $n \geq n_{0}$, define the stopping time

$$
\xi_{n}=\inf \{t \geq 0 \mid \max \{x(t), y(t)\} \geq n\} .
$$

Note $\xi_{n}$ is monotonically increasing and hence has a (finite or infinite) limit. Denote the limit by $\xi_{\infty}$. For any $n$ sufficiently large, we have $\xi_{n} \geq \tau_{n}$, where $\tau_{n}$ is defined by (2.2). By Theorem 2.1, we have $\tau_{\infty}=\infty$, then $\xi_{\infty}=\infty$. The local martingale property implies that $\mathbb{E}\left[M_{v_{1}}\left(t \wedge \xi_{n}\right)\right]=0$. That is, for any $t \geq 0$

$$
\begin{aligned}
\mathbb{E}\left[e^{\kappa\left(t \wedge \xi_{n}\right)} V_{1}\left(x\left(t \wedge \xi_{n}\right), y\left(t \wedge \xi_{n}\right)\right)\right]= & \mathbb{E}\left[V_{1}\left(x_{0}, y_{0}\right)\right] \\
& +\mathbb{E} \int_{0}^{t \wedge \xi_{n}} \mathcal{L}\left[e^{\kappa s} V_{1}(x(s), y(s))\right] \mathrm{d} s .
\end{aligned}
$$

From the definition of $\xi_{n}$, we have $e^{\kappa\left(t \wedge \xi_{n}\right)}\left(1+x\left(t \wedge \xi_{n}\right)+c y\left(t \wedge \xi_{n}\right)\right)^{\theta}$ is monotonically increasing in $n$. Let $n \rightarrow \infty$, we obtain

$$
e^{\kappa\left(t \wedge \xi_{n}\right)}\left(1+x\left(t \wedge \xi_{n}\right)+c y\left(t \wedge \xi_{n}\right)\right)^{\theta} \uparrow e^{\kappa t}(1+x(t)+c y(t))^{\theta} \quad \text { a.s. }
$$

By the monotone convergence theorem,

$$
\mathbb{E}\left[e^{\kappa\left(t \wedge \xi_{n}\right)} V_{1}\left(x\left(t \wedge \xi_{n}\right), y\left(t \wedge \xi_{n}\right)\right)\right] \rightarrow \mathbb{E}\left[e^{\kappa t} V_{1}(x(t), y(t))\right], \quad \text { as } n \rightarrow \infty .
$$

By (3.5) and the dominated convergence theorem,

$$
\mathbb{E} \int_{0}^{t \wedge \xi_{n}} \mathcal{L}\left[e^{\kappa s} V_{1}(x(s), y(s))\right] \mathrm{d} s \rightarrow \mathbb{E} \int_{0}^{t} \mathcal{L}\left[e^{\kappa s} V_{1}(x(s), y(s))\right] \mathrm{d} s, \quad \text { as } n \rightarrow \infty .
$$

Therefore, letting $n \rightarrow \infty$ in (3.6) yields

$$
\mathbb{E}\left[e^{\kappa t} V_{1}(x(t), y(t))\right]=\mathbb{E}\left[V_{1}\left(x_{0}, y_{0}\right)\right]+\mathbb{E} \int_{0}^{t} \mathcal{L}\left[e^{\kappa s} V_{1}(x(s), y(s))\right] \mathrm{d} s .
$$

Step 3. We complete the proof. By (3.5) and (3.7) we have

$$
e^{\kappa t} \mathbb{E}\left[(1+x(t)+c y(t))^{\theta}\right] \leq \mathbb{E}\left[\left(1+x_{0}+c y_{0}\right)^{\theta}\right]+\frac{L_{3}(c, \theta)}{\kappa} e^{\kappa t} .
$$

Then

$$
\mathbb{E}\left[(1+x(t)+c y(t))^{\theta}\right] \leq \mathbb{E}\left[\left(1+x_{0}+c y_{0}\right)^{\theta}\right] e^{-\kappa t}+\frac{L_{3}(c, \theta)}{\kappa}
$$


Letting $t \rightarrow \infty$, we obtain

$$
\limsup _{t \rightarrow \infty} \mathbb{E}\left[(1+x(t)+c y(t))^{\theta}\right] \leq \frac{L_{3}(c, \theta)}{\kappa}=: L(c, \theta) .
$$

The proof is complete.

The positivity of $y(t)$ implies the follow result directly.

Corollary 3.4 For any $\theta \in\left(0,1+2 \delta / \sigma_{1}^{2}\right)$ and $c>[\rho / \eta-\mu]^{+}$,

$$
\limsup _{t \rightarrow \infty} \mathbb{E}\left[(1+x(t))^{\theta}\right] \leq L(c, \theta),
$$

where $L(c, \theta)$ is defined in Theorem 3.3.

Due to the inequality direction in the theory of probability it is difficult to find the lower bound of the moment of $x(t)$. Alternatively, we try to look for the upper bound of the moment of $1 / x(t)$. Thus we get the following result.

Theorem 3.5 For any fixed $\theta>0$, there exists a positive constant $\bar{L}$ such that

$$
\limsup _{t \rightarrow \infty} \mathbb{E}\left[x^{-\theta}(t)\right] \leq \bar{L}
$$

Proof. Let $V_{2}(x)=\left(1+\frac{1}{x}\right)^{\theta}, \forall x>0$. Choosing a positive constant $\kappa$ and applying the Itô formula we obtain that

$$
M_{v_{2}}(t):=e^{\kappa t} V_{2}(x(t))-V_{2}\left(x_{0}\right)-\int_{0}^{t} \mathcal{L}_{x}\left[e^{\kappa s} V_{2}(x(s))\right] \mathrm{d} s
$$

is a local martingale, where

$$
\begin{aligned}
& \mathcal{L}_{x}\left[e^{\kappa t} V_{2}(x)\right] \\
& \begin{array}{r}
:=\theta e^{\kappa t}\left(1+\frac{1}{x}\right)^{\theta-2}\left[-\frac{\sigma}{x^{3}}-\left(\sigma-\delta-\frac{\theta+1}{2} \sigma_{1}^{2}-\frac{\kappa}{\theta}\right) \frac{1}{x^{2}}+\left(\delta+\sigma_{1}^{2}+\frac{2 \kappa}{\theta}\right) \frac{1}{x}\right. \\
\left.-\frac{\rho y}{x(\eta+y)}+\frac{\mu y}{x}-\frac{\rho y}{x^{2}(\eta+y)}+\mu \frac{y}{x^{2}}+\frac{\kappa}{\theta}\right] \\
\leq \theta e^{\kappa t}\left(1+\frac{1}{x}\right)^{\theta-2}\left[-\frac{\sigma}{x^{3}}-\left(\sigma-\delta-\frac{\theta+1}{2} \sigma_{1}^{2}-\frac{\kappa}{\theta}\right) \frac{1}{x^{2}}+\left(\delta+\sigma_{1}^{2}+\frac{2 \kappa}{\theta}\right) \frac{1}{x}\right. \\
\left.+\frac{\kappa}{\theta}\right]+2 \mu \theta e^{\kappa t}\left(1+\frac{1}{x}\right)^{\theta} y .
\end{array}
\end{aligned}
$$

Due to Young's inequality, one observes that

$$
\begin{aligned}
2 \mu\left(1+\frac{1}{x}\right)^{\theta} y & \leq \frac{2 \mu \theta}{\theta+\frac{1}{2}}\left(1+\frac{1}{x}\right)^{\theta+\frac{1}{2}}+\frac{\mu}{\theta+\frac{1}{2}} y^{2 \theta+1} \\
& =\frac{2 \mu \theta}{\theta+\frac{1}{2}}\left(1+\frac{1}{x}\right)^{\theta-2}\left(1+\frac{1}{x}\right)^{\frac{5}{2}}+\frac{2 \mu}{2 \theta+1} y^{2 \theta+1} \\
& \leq 8 \mu\left(1+\frac{1}{x}\right)^{\theta-2}\left(1+\frac{1}{x^{\frac{5}{2}}}\right)+\frac{2 \mu}{2 \theta+1} y^{2 \theta+1}
\end{aligned}
$$


in the last inequality, we have used the fact that $\left(1+\frac{1}{x}\right)^{\frac{5}{2}} \leq 2^{\frac{3}{2}}\left(1+\frac{1}{x^{\frac{5}{2}}}\right) \leq 4\left(1+\frac{1}{x^{\frac{5}{2}}}\right)$. This implies that

$$
\begin{aligned}
& \mathcal{L}_{x}\left[e^{\kappa t} V_{2}(x)\right] \\
& \leq \theta e^{\kappa t}\left(1+\frac{1}{x}\right)^{\theta-2}\left[-\frac{\sigma}{x^{3}}+\frac{8 \mu}{x^{\frac{5}{2}}}-\left(\sigma-\delta-\frac{\theta+1}{2} \sigma_{1}^{2}-\frac{\kappa}{\theta}\right) \frac{1}{x^{2}}\right. \\
& \left.+\left(\delta+\sigma_{1}^{2}+\frac{2 \kappa}{\theta}\right) \frac{1}{x}+\frac{\kappa}{\theta}+8 \mu\right]+\frac{2 \mu \theta}{2 \theta+1} e^{\kappa t} y^{2 \theta+1} \\
& \leq e^{\kappa t}\left(L_{4}+\frac{2 \mu \theta}{2 \theta+1} y^{2 \theta+1}\right) \text {, }
\end{aligned}
$$

where

$$
\begin{aligned}
L_{4}=\sup _{x \in \mathbb{R}_{+}}\left\{\theta\left(1+\frac{1}{x}\right)^{\theta-2}\right. & {\left[-\frac{\sigma}{x^{3}}+\frac{8 \mu}{x^{\frac{5}{2}}}-\left(\sigma-\delta-\frac{\theta+1}{2} \sigma_{1}^{2}-\frac{\kappa}{\theta}\right) \frac{1}{x^{2}}\right.} \\
& \left.\left.+\left(\delta+\sigma_{1}^{2}+\frac{2 \kappa}{\theta}\right) \frac{1}{x}+\frac{\kappa}{\theta}+8 \mu\right]\right\} .
\end{aligned}
$$

Let $n_{0}>0$ be sufficiently large for the initial value $x_{0}$ lying within the interval $\left(1 / n_{0}, n_{0}\right)$. For any $n \geq n_{0}$, define the stopping time

$$
\tilde{\xi}_{n}=\inf \{t \geq 0, x(t) \leq 1 / n\} .
$$

Note $\tilde{\xi}_{n}$ is monotonically increasing and hence has a (finite or infinite) limit. Denote the limit by $\tilde{\xi}_{\infty}$. For any $n$ sufficiently large, we have $\tilde{\xi}_{n} \geq \tau_{n}$, where $\tau_{n}$ is defined by $(2.2)$. By Theorem 2.1, we have $\tau_{\infty}=\infty$, so $\tilde{\xi}_{\infty}=\infty$. The local martingale property implies that $\mathbb{E}\left[M_{v_{2}}\left(t \wedge \tilde{\xi}_{n}\right)\right]=0$. That is, for any $t \geq 0$

$$
\mathbb{E}\left[e^{\kappa\left(t \wedge \tilde{\xi}_{n}\right)} V_{2}\left(x\left(t \wedge \tilde{\xi}_{n}\right)\right)\right]=\mathbb{E}\left[V_{2}\left(x_{0}\right)\right]+\mathbb{E} \int_{0}^{t \wedge \tilde{\xi}_{n}} \mathcal{L}_{x}\left[e^{\kappa s} V_{2}(x(s))\right] \mathrm{d} s .
$$

From the definition of $\tilde{\xi}_{n}$, we have $e^{\kappa\left(t \wedge \tilde{\xi}_{n}\right)}\left(1+\frac{1}{x\left(t \wedge \tilde{\xi}_{n}\right)}\right)^{\theta}$ is monotonically increasing. Letting $n \rightarrow \infty$ yields

$$
e^{\kappa\left(t \wedge \tilde{\xi}_{n}\right)}\left(1+\frac{1}{x\left(t \wedge \tilde{\xi}_{n}\right)}\right)^{\theta} \uparrow e^{\kappa t}\left(1+\frac{1}{x(t)}\right)^{\theta} \quad \text { a.s. }
$$

By the monotone convergence theorem one notices that as $n \rightarrow \infty$

$$
\mathbb{E}\left[e^{\kappa\left(t \wedge \tilde{\xi}_{n}\right)} V_{2}\left(x\left(t \wedge \tilde{\xi}_{n}\right)\right)\right] \rightarrow \mathbb{E}\left[e^{\kappa t} V_{2}(x(t))\right] .
$$

Noting that $\mathbb{E}\left(y^{2 \theta+1}(t)\right)$ is bounded uniformly with respect to $t \in[0, \infty)$, by the Fubini theorem and (3.10), we obtain

$$
\begin{aligned}
\mathbb{E} \int_{0}^{t} \mathcal{L}\left[e^{\kappa s} V_{2}(x(s))\right] \mathrm{d} s & \leq \mathbb{E} \int_{0}^{t} e^{\kappa s}\left(L_{4}+\frac{2 \mu \theta}{2 \theta+1} y^{2 \theta+1}(s)\right) \mathrm{d} s \\
& =\mathbb{E} \int_{0}^{t} L_{4} e^{\kappa s} \mathrm{~d} s+\frac{2 \mu \theta}{2 \theta+1} \int_{0}^{t} e^{\kappa s} \mathbb{E}\left(y^{2 \theta+1}(s)\right) \mathrm{d} s<\infty .
\end{aligned}
$$


Using the dominated convergence theorem implies that as $n \rightarrow \infty$

$$
\mathbb{E} \int_{0}^{t \wedge \tilde{\xi}_{n}} \mathcal{L}\left[e^{\kappa s} V_{2}(x(s))\right] \mathrm{d} s \rightarrow \mathbb{E} \int_{0}^{t} \mathcal{L}\left[e^{\kappa s} V_{2}(x(s))\right] \mathrm{d} s .
$$

Therefore, letting $n \rightarrow \infty$ yields

$$
\mathbb{E}\left[e^{\kappa t} V_{2}(x(t))\right]=\mathbb{E}\left[V_{2}\left(x_{0}\right)\right]+\mathbb{E} \int_{0}^{t} \mathcal{L}\left[e^{\kappa s} V_{2}(x(s))\right] \mathrm{d} s .
$$

This together with Theorem 3.2 implies

$$
\begin{aligned}
& e^{\kappa t} \mathbb{E}\left[\left(1+\frac{1}{x(t)}\right)^{\theta}\right] \\
& \leq\left(1+\frac{1}{x_{0}}\right)^{\theta}+\mathbb{E} \int_{0}^{t} e^{\kappa s}\left(L_{4}+\frac{2 \mu \theta}{2 \theta+1} y^{2 \theta+1}(s)\right) \mathrm{d} s \leq\left(1+\frac{1}{x_{0}}\right)^{\theta}+\frac{L_{5}}{\kappa} e^{\kappa t},
\end{aligned}
$$

where $L_{5}:=L_{4}+\frac{2 \mu \theta}{2 \theta+1} \sup _{t \geq 0} \mathbb{E}\left(y^{2 \theta+1}(t)\right)<\infty$. Hence

$$
\mathbb{E}\left[\left(1+\frac{1}{x(t)}\right)^{\theta}\right] \leq\left(1+\frac{1}{x_{0}}\right)^{\theta} e^{-\kappa t}+\frac{L_{5}}{\kappa} .
$$

We therefore obtain

$$
\limsup _{t \rightarrow \infty} \mathbb{E}\left[x^{-\theta}(t)\right] \leq \limsup _{t \rightarrow \infty} \mathbb{E}\left[\left(1+\frac{1}{x(t)}\right)^{\theta}\right] \leq \frac{L_{5}}{\kappa}=: \bar{L} .
$$

The proof is complete.

\section{Existence and uniqueness of invariant measure}

This section is devoted to analyze the invariant measure. Define function

$$
f_{2}(y)=\frac{\rho y}{\eta+y}-\mu y, \quad y \geq 0 .
$$

Similar to the analysis of the function $f_{1}(y)$ in Theorem 3.3, we obtain

(i) If $\rho \leq \mu \eta, f_{2}(y)<0, \forall y>0$.

(ii) If $\rho>\mu \eta, f_{2}(y) \leq(\sqrt{\rho}-\sqrt{\mu \eta})^{2}, \forall y>0$. 
This implies that for any $y>0, f_{2}(y) \leq[(\sqrt{\rho}-\sqrt{\mu \eta}) \vee 0]^{2}$. We now introduce a new auxiliary process $\varphi(t)$ with respect to $x(t)$ described by

$$
\left\{\begin{array}{l}
\mathrm{d} \varphi(t)=\left[\sigma-\left(\delta-h^{2}\right) \varphi(t)\right] \mathrm{d} t+\sigma_{1} \varphi(t) \mathrm{d} B_{1}(t), \\
\varphi(0)=x_{0}>0,
\end{array}\right.
$$

where

$$
h:=(\sqrt{\rho}-\sqrt{\mu \eta}) \vee 0 .
$$

If $\delta-h^{2}>0$, by solving the Fokker-Planck equation (see details in [9, p.1065]), the process $\varphi(t)$ has a unique stationary distribution $\nu(\cdot)$, which is the inverse Gamma distribution with parameter

$$
a_{1}=\frac{2\left(\delta-h^{2}\right)}{\sigma_{1}^{2}}+1, \quad b_{1}=\frac{2 \sigma}{\sigma_{1}^{2}} .
$$

And probability density of $\nu(\cdot)$ is

$$
f^{*}(x)=\frac{b_{1}^{a_{1}}}{\Gamma\left(a_{1}\right)} x^{-\left(a_{1}+1\right)} e^{-\frac{b_{1}}{x}}, \quad x>0 .
$$

With a notation abuse slightly, we write $\varphi \sim I G\left(a_{1}, b_{1}\right)$. For any $p>0$, by the strong ergodicity we deduce that

$$
\lim _{t \rightarrow \infty} \frac{1}{t} \int_{0}^{t} \varphi^{p}(s) \mathrm{d} s=\int_{0}^{\infty} x^{p} f^{*}(x) \mathrm{d} x=: M_{p} \quad \text { a.s. }
$$

Especially, if $p=1, \quad M_{1}=\frac{\sigma}{\delta-h^{2}}$. Moreover, the stationary distribution of $\frac{1}{\varphi(t)}$ is the Gamma distribution with parameter $a_{1}$ and $b_{1}$, see details in [11, p.189]. Therefore, changing the variable by the Itô formula, noting that the mean of Gamma distribution is $a_{1} / b_{1}$, and by the strong ergodicity we arrive at

$$
\lim _{t \rightarrow \infty} \frac{1}{t} \ln \varphi(t)=\lim _{t \rightarrow \infty} \frac{1}{t} \int_{0}^{t}\left(\frac{\sigma}{\varphi(s)}-\delta+h^{2}-\frac{\sigma_{1}^{2}}{2}\right) d s+\sigma_{1} \lim _{t \rightarrow \infty} \frac{B_{1}(t)}{t}=0,
$$

By virtue of the comparison theorem it follows that $0<x(t) \leq \varphi(t)$ for all $t \geq 0$ a.s. This implies,

$$
\limsup _{t \rightarrow \infty} \frac{1}{t} \ln x(t) \leq 0 \quad \text { a.s. }
$$

Furthermore, we derive the following result from (4.3). 
Lemma 4.1 If $\delta-h^{2}>0$, then we have

$$
\limsup _{t \rightarrow \infty} \frac{1}{t} \int_{0}^{t} x^{p}(s) \mathrm{d} s \leq M_{p}, \quad \forall p>0, \quad \text { a.s. }
$$

Moreover,

$$
\limsup _{t \rightarrow \infty} \frac{1}{t} \int_{0}^{t} x(s) \mathrm{d} s \leq \frac{\sigma}{\delta-h^{2}} \quad \text { a.s. }
$$

To obtain more properties of $x(t)$, we go a further step to consider the equation on the boundary $y(t)=0$

$$
\left\{\begin{array}{l}
\mathrm{d} z(t)=(\sigma-\delta z(t)) \mathrm{d} t+\sigma_{1} z(t) \mathrm{d} B_{1}(t), \quad \forall t \geq t_{0} \\
z\left(t_{0}\right)=x\left(t_{0}\right)
\end{array}\right.
$$

where $t_{0} \geq 0$ will be chosen latter. By solving the Fokker-Planck equation (see details in $[9, \mathrm{p} .1065])$, the process $z(t)$ has a unique stationary distribution $\mu(\cdot)$, and obeys the inverse Gamma distribution with parameter

$$
a_{2}=\frac{2 \delta}{\sigma_{1}^{2}}+1, \quad b_{2}=\frac{2 \sigma}{\sigma_{1}^{2}} .
$$

And probability density of $\mu(\cdot)$ is

$$
p^{*}(x)=\frac{\left(b_{2}\right)^{a_{2}}}{\Gamma\left(a_{2}\right)} x^{-\left(a_{2}+1\right)} e^{-\frac{b_{2}}{x}}, \quad x>0 .
$$

With a notation abuse slightly, we write $z \sim I G\left(a_{2}, b_{2}\right)$.

In the following, we will reveal the long-time behavior of the tumor cells and the effector cells, if the intensity of the noise $\sigma_{2}$ is large sufficiently.

Theorem 4.2 If $\lambda_{1}:=\frac{\sigma_{2}^{2}}{2}-\alpha>0$, then we have

$$
\limsup _{t \rightarrow \infty} \frac{\ln y(t)}{t} \leq-\lambda_{1}
$$

and the distribution of $x(t)$ converges to a unique invariant probability measure $\pi_{1}(\cdot)$ as $t \rightarrow \infty$.

Proof. By virtue of the auxiliary process $\psi(t)$ defined by (3.1), changing the variable by the Itô formula leads to

$$
\limsup _{t \rightarrow \infty} \frac{1}{t} \ln y(t) \leq \limsup _{t \rightarrow \infty} \frac{1}{t} \ln \psi(t) \leq-\lambda_{1} \quad \text { a.s. }
$$


For any $\varepsilon>0$, let $T>0$ be sufficiently large satisfying $\max \left\{\mu, \frac{\rho}{\eta}\right\}<\frac{\varepsilon \lambda_{1}}{2} \exp \left(\frac{\lambda_{1} T}{2}\right)$ such that $\mathbb{P}\left(\Omega_{\varepsilon}\right)>1-\varepsilon$, where $\Omega_{\varepsilon}:=\left\{y(t) \leq \exp \left(-\frac{\lambda_{1} t}{2}\right), \forall t \geq T\right\}$.

Case (1). Suppose $\rho \leq \mu \eta$, then $f_{2}(y)=\frac{\rho y}{\eta+y}-\mu y \leq 0$. Fix a constant $t_{0} \geq T$, by the comparison theorem, we have $\mathbb{P}\left\{z(t) \geq x(t), \quad \forall t \geq t_{0}\right\}=1$. By the Itô formula, we deduce that for almost all $\omega \in \Omega_{\varepsilon}, \forall t \geq t_{0}$,

$$
\begin{aligned}
0 \leq \ln z(t)-\ln x(t) & =\sigma \int_{t_{0}}^{t}\left(\frac{1}{z(s)}-\frac{1}{x(s)}\right) \mathrm{d} s-\int_{t_{0}}^{t} \frac{\rho y(s)}{\eta+y(s)} \mathrm{d} s+\mu \int_{t_{0}}^{t} y(s) \mathrm{d} s \\
& \leq \mu \int_{t_{0}}^{t} \exp \left(-\frac{\lambda_{1} s}{2}\right) \mathrm{d} s \\
& =\frac{2 \mu}{\lambda_{1}}\left[\exp \left(-\frac{\lambda_{1} t_{0}}{2}\right)-\exp \left(-\frac{\lambda_{1} t}{2}\right)\right] \leq \frac{2 \mu}{\lambda_{1}} \exp \left(-\frac{\lambda_{1} T}{2}\right)<\varepsilon .
\end{aligned}
$$

Case (2). Suppose $\rho>\mu \eta$, then $f_{2}^{\prime}(0)>0$. Due to the continuity of $f_{2}(y)$ at $y=0$, one may choose a positive constant $\hat{y}$ such that for any $0<y<\hat{y}, f_{2}(y)>0$. Choose a constant $t_{0} \geq T$ satisfying $\exp \left(-\frac{\lambda_{1} t_{0}}{2}\right) \leq \hat{y}$. By the comparison theorem, $x(t) \geq z(t), \forall t \geq t_{0}$, for almost all $\omega \in \Omega_{\varepsilon}$. By the Itô formula we deduce that, for almost all $\omega \in \Omega_{\varepsilon}, \forall t \geq t_{0}$,

$$
\begin{aligned}
0 \leq \ln x(t)-\ln z(t) & =\sigma \int_{t_{0}}^{t}\left(\frac{1}{x(s)}-\frac{1}{z(s)}\right) \mathrm{d} s+\int_{t_{0}}^{t} \frac{\rho y(s)}{\eta+y(s)} \mathrm{d} s-\mu \int_{t_{0}}^{t} y(s) \mathrm{d} s \\
& \leq \frac{\rho}{\eta} \int_{t_{0}}^{t} y(s) \mathrm{d} s \leq \frac{\rho}{\eta} \int_{t_{0}}^{t} \exp \left(-\frac{\lambda_{1} s}{2}\right) \mathrm{d} s \\
& =\frac{2 \rho}{\lambda_{1} \eta}\left[\exp \left(-\frac{\lambda_{1} t_{0}}{2}\right)-\exp \left(-\frac{\lambda_{1} t}{2}\right)\right] \leq \frac{2 \rho}{\lambda_{1} \eta} \exp \left(-\frac{\lambda_{1} T}{2}\right)<\varepsilon .
\end{aligned}
$$

Therefore

$$
\mathbb{P}\{|\ln z(t)-\ln x(t)|>\varepsilon\} \leq 1-\mathbb{P}\left(\Omega_{\varepsilon}\right)<\varepsilon, \quad \forall t \geq t_{0},
$$

which implies that $\ln x(t)$ converges weakly to $\ln z(t)$. Let $\pi_{1}^{*}(\cdot)$ be the invariant measure of $\ln z(t)$. In order to show that the distribution of $x(t)$ converges to a probability measure $\pi_{1}(\cdot)$, we only need to prove that the distribution of $\ln x(t)$ converges to $\pi_{1}^{*}(\cdot)$. Let $\mathcal{P}(\mathbb{R})$ represents the family of all probability measures on $\mathbb{R}$. For any $\mathbb{P}_{1}, \mathbb{P}_{2} \in \mathcal{P}(\mathbb{R})$, define the distance as in $[26$, p.212]

$$
\mathrm{d}_{\mathbb{L}}\left(\mathbb{P}_{1}, \mathbb{P}_{2}\right)=\sup _{f \in \mathbb{L}}\left|\int_{\mathbb{R}} f(x) \mathbb{P}_{1}(\mathrm{~d} x)-\int_{\mathbb{R}} f(x) \mathbb{P}_{2}(\mathrm{~d} x)\right|,
$$

where

$$
\mathbb{L}=\{f: \mathbb{R} \rightarrow \mathbb{R}:|f(x)-f(y)| \leq|x-y| \text { and }|f(\cdot)| \leq 1\}
$$


By the Portmanteau theorem, we need to prove that for any $f \in \mathbb{L}$,

$$
\mathbb{E} f(\ln x(t)) \rightarrow \bar{f}:=\int_{\mathbb{R}} f(x) \pi_{1}^{*}(\mathrm{~d} x)=\int_{0}^{\infty} f(\ln x) \pi_{1}(\mathrm{~d} x) .
$$

Since the diffusion is nondegenerate, it is well known that as $t \rightarrow \infty$ the distribution of $\ln z(t)$ converges to the unique stationary distribution $\pi_{1}^{*}(\cdot)$, namely,

$$
\lim _{t \rightarrow \infty} \mathbb{E} f(\ln z(t))=\bar{f}
$$

We now compute

$$
\begin{aligned}
\mid \mathbb{E} f & (\ln x(t))-\bar{f} \mid \\
& \leq|\mathbb{E} f(\ln x(t))-\mathbb{E} f(\ln z(t))|+|\mathbb{E} f(\ln z(t))-\bar{f}| \\
& \leq \mathbb{E}\left[|f(\ln x(t))-f(\ln z(t))| \mathbf{I}_{\{|\ln x(t)-\ln z(t)| \leq \varepsilon\}}\right] \\
& +\mathbb{E}\left[|f(\ln x(t))-f(\ln z(t))| \mathbf{I}_{\{|\ln x(t)-\ln z(t)|>\varepsilon\}}\right]+|\mathbb{E} f(\ln z(t))-\bar{f}| \\
& \left.\leq \varepsilon \mathbb{E}\left[\mathbf{I}_{\{|\ln x(t)-\ln z(t)| \leq \varepsilon\}}\right]+2 \mathbb{E}\left[\mathbf{I}_{\{|\ln x(t)-\ln z(t)|>\varepsilon}\right\}\right]+|\mathbb{E} f(\ln z(t))-\bar{f}| \\
& =\varepsilon+2 \mathbb{P}\{|\ln z(t)-\ln x(t)|>\varepsilon\}+|\mathbb{E} f(\ln z(t))-\bar{f}| .
\end{aligned}
$$

This, together with (4.9) and (4.10), yields

$$
\limsup _{t \rightarrow \infty}|\mathbb{E} f(\ln x(t))-\bar{f}|=0 .
$$

The proof is therefore complete.

Remark 4.3 Theorem 4.2 predicts that when the stochastic perturbation for tumor cells is strong enough the tumor is going to die out at exponential rate while the distribution of effector cells converges to a steady state $\pi_{1}$. It is clear that $\sigma_{2}$ is a critical parameter to eliminate the tumor cells and let the effector cells tend to a steady state in stochastic version. This implies that the dynamics of the stochastic model (1.2) are completely different from those of the deterministic model (1.1) in [22]. .

Recall the auxiliary process $\psi(t)$ defined by (3.1). We have proved that $\alpha<$ $\sigma_{2}^{2} / 2$ implies $\limsup \psi(t)=0$ a.s due to (4.8). If $\alpha>\sigma_{2}^{2} / 2$, by solving the FokkerPlanck equation (see details in $[11, \mathrm{p} .189]$ ), the process $\psi(t)$ has a unique stationary distribution $\lambda(\cdot)$, and obeys the Gamma distribution with parameter

$$
a_{3}=\frac{2 \alpha}{\sigma_{2}^{2}}-1, \quad b_{3}=\frac{2 \beta}{\sigma_{2}^{2}} .
$$

The probability density of $\lambda(\cdot)$ is

$$
g^{*}(x)=\frac{\left(b_{3}\right)^{a_{3}}}{\Gamma\left(a_{3}\right)} x^{a_{3}-1} e^{-b_{3} x}, \quad x>0 .
$$


With a notation abuse slightly, we write $\psi \sim G\left(a_{3}, b_{3}\right)$. For any $p>0$, by the strong ergodicity we derive that

$$
\lim _{t \rightarrow \infty} \frac{1}{t} \int_{0}^{t} \psi^{p}(s) \mathrm{d} s=\int_{0}^{\infty} x^{p} g^{*}(x) \mathrm{d} x=: \bar{M}_{p} \quad \text { a.s. }
$$

In particular, if $p=1$, we have $\bar{M}_{1}=\frac{1}{\beta}\left(\alpha-\frac{\sigma_{2}^{2}}{2}\right)$. Therefore, using the Itô formula implies

$$
\lim _{t \rightarrow \infty} \frac{1}{t} \ln \psi(t)=\lim _{t \rightarrow \infty} \frac{1}{t} \int_{0}^{t}\left(\alpha-\frac{\sigma_{2}^{2}}{2}-\beta \psi(s)\right) \mathrm{d} s+\sigma_{2} \lim _{t \rightarrow \infty} \frac{B_{2}(t)}{t}=0 .
$$

By virtue of the comparison theorem it follows that $0<y(t) \leq \psi(t)$ for all $t \geq 0$ a.s. One observes that

$$
\limsup _{t \rightarrow \infty} \frac{1}{t} \ln y(t) \leq 0 \quad \text { a.s. }
$$

Furthermore, we have the following result from (4.12).

Lemma 4.4 If $\alpha>\frac{\sigma_{2}^{2}}{2}$, then the property

$$
\limsup _{t \rightarrow \infty} \frac{1}{t} \int_{0}^{t} y^{p}(s) \mathrm{d} s \leq \bar{M}_{p}, \quad \forall p>0, \quad \text { a.s. }
$$

holds. Moreover,

$$
\limsup _{t \rightarrow \infty} \frac{1}{t} \int_{0}^{t} y(s) \mathrm{d} s \leq \frac{1}{\beta}\left(\alpha-\frac{\sigma_{2}^{2}}{2}\right) \quad \text { a.s. }
$$

In order to investigate the probability law for the small noises we prepare two lemmas.

Lemma 4.5 If $\alpha>\frac{\sigma_{2}^{2}}{2}$, then the property

$$
\limsup _{t \rightarrow \infty} \frac{1}{t} \int_{0}^{t} \frac{1}{x(s)} \mathrm{d} s \leq \lambda_{2}
$$

holds, where $\lambda_{2}:=\frac{1}{\sigma}\left[\frac{\mu}{\beta}\left(\alpha-\frac{\sigma_{2}^{2}}{2}\right)+\delta+\frac{\sigma_{1}^{2}}{2}\right]$.

Proof. For any $\left(x_{0}, y_{0}\right) \in \mathbb{R}_{+}^{2}$, using the fact $0<y(t) \leq \psi(t)$ a.s. and the Itô formula, we have

$$
\begin{aligned}
\frac{1}{t} \ln x(t) & =\frac{1}{t} \int_{0}^{t}\left(\frac{\sigma}{x(s)}+\frac{\rho y(s)}{\eta+y(s)}-\mu y(s)\right) \mathrm{d} s-\delta-\frac{\sigma_{1}^{2}}{2}+\frac{\ln x_{0}}{t}+\frac{\sigma_{1} B_{1}(t)}{t} \\
& \geq \frac{1}{t} \int_{0}^{t} \frac{\sigma}{x(s)} \mathrm{d} s-\frac{1}{t} \int_{0}^{t} \mu \psi(s) \mathrm{d} s-\delta-\frac{\sigma_{1}^{2}}{2}+\frac{\ln x_{0}}{t}+\frac{\sigma_{1} B_{1}(t)}{t} .
\end{aligned}
$$


Letting $t \rightarrow \infty$, by the strong ergodicity, (4.5) and (4.12) we deduce that

$$
\begin{aligned}
\limsup _{t \rightarrow \infty} \frac{1}{t} \int_{0}^{t} \frac{1}{x(s)} \mathrm{d} s & \leq \frac{1}{\sigma}\left(\limsup _{t \rightarrow \infty} \frac{1}{t} \int_{0}^{t} \mu \psi(s) \mathrm{d} s+\delta+\frac{\sigma_{1}^{2}}{2}\right) \\
& =\frac{1}{\sigma}\left[\frac{\mu}{\beta}\left(\alpha-\frac{\sigma_{2}^{2}}{2}\right)+\delta+\frac{\sigma_{1}^{2}}{2}\right] .
\end{aligned}
$$

The proof is complete.

Lemma 4.6 If $\delta>h^{2}$ and $\alpha-\frac{\sigma_{2}^{2}}{2}-\frac{\sigma}{\delta-h^{2}}>0$, then the inequality

$$
\liminf _{t \rightarrow \infty} \frac{1}{t} \int_{0}^{t} y(s) \mathrm{d} s \geq \lambda_{3}
$$

holds, where $\lambda_{3}:=\frac{1}{\beta}\left(\alpha-\frac{\sigma_{2}^{2}}{2}-\frac{\sigma}{\delta-h^{2}}\right)$.

Proof. For any $\left(x_{0}, y_{0}\right) \in \mathbb{R}_{+}^{2}$, since $0<x(t) \leq \varphi(t)$ a.s., an application of the Itô formula yields

$$
\begin{aligned}
\frac{1}{t} \ln y(t) & =\alpha-\frac{\sigma_{2}^{2}}{2}-\frac{1}{t} \int_{0}^{t} \beta y(s) \mathrm{d} s-\frac{1}{t} \int_{0}^{t} x(s) \mathrm{d} s+\frac{\ln y_{0}}{t}+\frac{\sigma_{2} B_{2}(t)}{t} \\
& \geq \alpha-\frac{\sigma_{2}^{2}}{2}-\frac{1}{t} \int_{0}^{t} \beta y(s) \mathrm{d} s-\frac{1}{t} \int_{0}^{t} \varphi(s) \mathrm{d} s+\frac{\ln y_{0}}{t}+\frac{\sigma_{2} B_{2}(t)}{t}
\end{aligned}
$$

Taking $t \rightarrow \infty$, by (4.3) and (4.14) we have

$$
\begin{aligned}
\liminf _{t \rightarrow \infty} \frac{1}{t} \int_{0}^{t} y(s) \mathrm{d} s & \geq \frac{1}{\beta}\left(\alpha-\frac{\sigma_{2}^{2}}{2}-\liminf _{t \rightarrow \infty} \frac{1}{t} \int_{0}^{t} \varphi(s) \mathrm{d} s\right) \\
& =\frac{1}{\beta}\left(\alpha-\frac{\sigma_{2}^{2}}{2}-\frac{\sigma}{\delta-h^{2}}\right) .
\end{aligned}
$$

The proof is complete.

Based on the above analysis we prove the existence and uniqueness of the invariant measure of the equation (1.2) under weak noises.

Theorem 4.7 If $\delta>h^{2}$ and $\alpha-\frac{\sigma_{2}^{2}}{2}-\frac{\sigma}{\delta-h^{2}}>0$, the solution $(x(t), y(t))$ of $(1.2)$ has a unique invariant measure $\pi_{2}(\cdot)$ with the support set $\mathbb{R}_{+}^{2}$.

Proof. For convenience, let $2 \zeta:=\left(\delta-h^{2}\right)\left(\alpha-\frac{\sigma_{2}^{2}}{2}\right)-\sigma$. Obviously, the given conditions imply $\zeta>0$. Furthermore, choose a constant $c>0$ small sufficiently such that

$$
c\left(\delta+\sigma_{1}^{2}\right) \leq \sigma \zeta .
$$


Define $U: \mathbb{R}_{+}^{2} \rightarrow \mathbb{R}_{+}$by

$$
U(x, y)=x+\frac{c}{x}+y^{2}+\left(\delta-h^{2}\right) \ln \left(1+\frac{1}{y}\right) .
$$

Computing $\mathcal{L} U(x, y)$ yields

$$
\begin{aligned}
\mathcal{L} U(x, y) & \\
= & \left(\sigma+\frac{\rho x y}{\eta+y}-\mu x y-\delta x\right)-c\left(\frac{\sigma}{x^{2}}+\frac{\rho y}{x(\eta+y)}-\frac{\mu y}{x}-\frac{\delta+\sigma_{1}^{2}}{x}\right)+\left[\left(2 \alpha+\sigma_{2}^{2}\right) y^{2}\right. \\
& \left.-2 \beta y^{3}-2 x y^{2}\right]+\left(\delta-h^{2}\right)\left[-\frac{\sigma_{2}^{2}}{2(y+1)^{2}}-\frac{\alpha-\sigma_{2}^{2}-x}{y+1}+\frac{\beta y}{y+1}\right] .
\end{aligned}
$$

Noting that the definition of $h$ in (4.2) and $\delta>h^{2}, \frac{\mu y}{x} \leq \frac{\sigma}{2 x^{2}}+\frac{\mu^{2}}{2 \sigma} y^{2}$, one observes that

$$
\begin{aligned}
\mathcal{L} U(x, y) \leq & {\left[\sigma-\left(\delta-h^{2}\right) x\right]-\frac{c \sigma}{2 x^{2}}+\frac{c\left(\delta+\sigma_{1}^{2}\right)}{x}+\left(2 \alpha+\sigma_{2}^{2}+\frac{c \mu^{2}}{2 \sigma}\right) y^{2} } \\
& -2 \beta y^{3}+\left(\delta-h^{2}\right)\left[-\frac{\sigma_{2}^{2}}{2(y+1)^{2}}-\frac{\alpha-\sigma_{2}^{2}-x}{y+1}+\frac{\beta y}{y+1}\right] .
\end{aligned}
$$

One can see that

$$
\lim _{x \rightarrow+\infty, y \rightarrow+\infty} \mathcal{L} U(x, y)=-\infty,
$$

which implies that there exist positive constants $H_{x}^{1}$ and $H_{y}^{1}$ such that

$$
\mathcal{L} U(x, y) \leq-\zeta, \quad \forall x \geq H_{x}^{1}, y \geq H_{y}^{1} .
$$

Moreover, we can show that

$$
\lim _{x \rightarrow 0+, y \rightarrow+\infty} \mathcal{L} V(x, y)=-\infty,
$$

which implies that there exist positive constants $h_{x}^{1}<H_{x}^{1}$ and $H_{y}^{2} \geq H_{y}^{1}$ such that

$$
\mathcal{L} U(x, y) \leq-\zeta, \quad 0<x \leq h_{x}^{1}, y \geq H_{y}^{2} .
$$

One goes a further step to notice that

$$
\lim _{y \rightarrow+\infty} \mathcal{L} U(x, y)=-\infty, \quad h_{x}^{1}<x<H_{x}^{1},
$$

which implies that there exists a positive constant $H_{y} \geq H_{y}^{2}$ such that

$$
\mathcal{L} U(x, y) \leq-\zeta, \quad h_{x}^{1}<x<H_{x}^{1}, y \geq H_{y} .
$$

Combing (4.21), (4.22) and (4.23) yields

$$
\mathcal{L} U(x, y) \leq-\zeta, \quad \forall x>0, y \geq H_{y} .
$$


One notices that

$$
\lim _{x \rightarrow 0+} \mathcal{L} U(x, y)=-\infty, \quad 0<y<H_{y},
$$

which implies that there exist a positive constant $h_{x}$ such that

$$
\mathcal{L} U(x, y) \leq-\zeta, \quad 0<x \leq h_{x}, 0<y<H_{y} .
$$

Note that

$$
\limsup _{x \rightarrow+\infty, y \rightarrow 0+} \mathcal{L} U(x, y) \leq-2 \zeta .
$$

which implies that there exist positive constants $H_{x}^{1}>h_{x}$ and $h_{y}^{1}<H_{y}$ such that

$$
\mathcal{L} U(x, y) \leq-\zeta, \quad H_{x}^{1} \leq x, 0<y \leq h_{y}^{1} .
$$

Once more, we can see that

$$
\lim _{x \rightarrow+\infty} \mathcal{L} U(x, y)=-\infty, \quad h_{y}^{1}<y<H_{y}
$$

which implies that there exists a positive constant $H_{x} \geq H_{x}^{1}$ such that

$$
\mathcal{L} U(x, y) \leq-\zeta, \quad H_{x} \leq x, h_{y}^{1}<y<H_{y} .
$$

Combing (4.26) and (4.27) yields

$$
\mathcal{L} U(x, y) \leq-\zeta, \quad H_{x} \leq x, 0<y<H_{y} .
$$

Due to (4.18), we know that for any $x>0$,

$$
-\frac{c \sigma}{2 x^{2}}+\frac{c\left(\delta+\sigma_{1}^{2}\right)}{x} \leq-\frac{c \sigma}{2}\left(\frac{1}{x}-\frac{\delta+\sigma_{1}^{2}}{\sigma}\right)^{2}+\frac{c\left(\delta+\sigma_{1}^{2}\right)^{2}}{2 \sigma} \leq \frac{c\left(\delta+\sigma_{1}^{2}\right)^{2}}{2 \sigma} \leq \frac{\zeta}{2}
$$

From the above inequality one observes that

$$
\limsup _{y \rightarrow 0+} \mathcal{L} U(x, y) \leq-\frac{3 \zeta}{2}, \quad \forall x>0,
$$

which implies that there exists a positive constant $h_{y}<H_{y}$ such that

$$
\mathcal{L} U(x, y) \leq-\zeta, \quad 0<x, 0<y \leq h_{y} .
$$

Therefore, (4.24), (4.25), (4.28) and (4.29) imply

$$
\mathcal{L} U(x, y) \leq-\zeta, \quad(x, y) \notin D=\left\{(x, y) \in \mathbb{R}_{+}^{2}: h_{x}<x<H_{x}, h_{y}<y<H_{y}\right\} .
$$

By [20, pp.106-122], $(x(t), y(t))$ is positive recurrent with respect to $D$. This together with the non-degeneracy of the diffusion coefficient yields that the solution $(x(t), y(t))$ of (1.2) has a unique invariant measure $\pi_{2}(\cdot)$.

Moreover, since the coefficients of (1.2) satisfy the conditions of [4, Corollaries 2.9 and $2.10]$, the support of the invariant measure $\pi_{2}(\cdot)$ is $\mathbb{R}_{+}^{2}$.

By [19, p.249] and [20, p.110, Theorem 4.2; p.114, Lemma 4.10], we have the following ergodicity result. 
Theorem 4.8 Under the conditions of Theorem 4.7, the following properties hold.

(i) For any $\pi_{2}$-integrable $f(x, y): \mathbb{R}_{+}^{2} \rightarrow \mathbb{R}$, we have

$$
\lim _{t \rightarrow \infty} \frac{1}{t} \int_{0}^{t} f(x(s), y(s)) \mathrm{d} s=\int_{\mathbb{R}_{+}^{2}} f(x, y) \pi_{2}(\mathrm{~d} x, \mathrm{~d} y) \quad \text { a.s. } \forall\left(x_{0}, y_{0}\right) \in \mathbb{R}_{+}^{2} .
$$

(ii) Let $\|\cdot\|$ var denote the total variation norm, for $(x, y) \in \mathbb{R}_{+}^{2}$, we have

$$
\lim _{t \rightarrow \infty}\left\|\mathbb{P}(t,(x, y), \cdot)-\pi_{2}(\cdot)\right\|=0, \quad \forall(x, y) \in \mathbb{R}_{+}^{2} .
$$

(iii) For any $\varepsilon>0$, there is $\zeta \in(0,1)$ such that

$$
\liminf _{t \rightarrow \infty} \mathbb{P}\left(t, x, y,\left[\zeta, \zeta^{-1}\right] \times\left[\zeta, \zeta^{-1}\right]\right)>1-\varepsilon .
$$

For a biological system the property (iii) of Theorem 4.8 is also called stochastically strong permanence.

Remark 4.9 Theorems 4.7 and 4.8 predict that under the small tumor noises the distribution of both tumor cells and effectors cells tends to a invariant measure $\pi_{2}$ as $t \rightarrow \infty$. This means that the tumor cells approach to a dormant steady state in stochastic version. The similar phenomenon was observed for the deterministic model (1.1) in [22].

\section{$5 \quad$ Examples and numerical simulations}

In this section, we mainly illustrate the effects of noise intensity on effector cells and tumor cells. We select the data in [22] and [33], see Table 4.1 below.

Table 4.1: The Significance and value of the parameters 


\begin{tabular}{lll}
\hline Parameter & Real value $/$ unit & Biological significance \\
\hline$a$ & $0.18 /$ day & the intrinsic growth rate of the TC \\
$b$ & $2.0 \times 10^{-9} /$ day & Reciprocal of environmental \\
& & capacity of TC \\
$s$ & $1.3 \times 10^{4} /$ piece $\times$ day & the normal rate of inflow into the \\
& & tumor site for EC \\
$d$ & $0.0412 /$ day & the coefficient of destruction and \\
& & migration of EC \\
$g$ & $2.019 \times 10^{7}$ piece & the positive constant in response \\
& & functional \\
$r_{1}$ & $0.1245 /$ day & $q=f K, K=\frac{k_{1}}{k_{2}+k_{3}+k_{-1}}$ \\
$r_{2}$ & $2.422 \times 10^{-10} /$ day $\times$ piece & $r_{1}=K k_{3}$ \\
\hline
\end{tabular}

where $f$ is the positive constant of response function, $k_{1}$ and $k_{-1}$ describe the rates of binding of EC to TC and detachment of EC from TC without damaging cells, $k_{2}$ is the rate at which EC-TC interactions irreversibly program TC for lysis, and $k_{3}$ is the rate at which EC-TC interactions inactivate EC. The non-dimensional treatment of the equation is done by selecting the order of magnitude scales $E_{0}$ and $T_{0}$ for the $E$ and $T$ cell populations, respectively, where $E_{0}=T_{0}=10^{6}$ cells [22, p.304]. Using the non-dimensionalization method in $[22$, p.304] yields coefficients in the model (1.2) as follows

$$
\begin{array}{lll}
\sigma=\frac{s}{r_{2} E_{0} T_{0}}=0.1181, & \rho=\frac{q}{r_{2} T_{0}}=1.131, & \mu=\frac{r_{1}}{r_{2}}=0.00311, \\
\delta=\frac{d}{r_{2} T_{0}}=0.3743, & \alpha=\frac{a}{r_{2} T_{0}}=1.636, & \eta=\frac{g}{T_{0}}=20.19, \\
\beta=\frac{a b}{r_{2}}=3.272 \times 10^{-3}, & x_{0}=5, & y_{0}=50 .
\end{array}
$$

Applying the Milstein method in Higham [18, p.539] yields the discrete equation as follows

$$
\left\{\begin{array}{l}
x_{k+1}=x_{k}+\left(\sigma+\frac{\rho x_{k} y_{k}}{\eta+y_{k}}-\mu x_{k} y_{k}-\delta x_{k}\right) \Delta t+\sigma_{1} x_{k} \sqrt{\Delta t} \xi_{k}+\frac{\sigma_{1}^{2} x_{k}^{2}}{2}\left(\xi_{k}^{2}-1\right) \Delta t, \\
y_{k+1}=y_{k}+\left(\alpha y_{k}-\beta y_{k}^{2}-x_{k} y_{k}\right) \Delta t+\sigma_{2} y_{k} \sqrt{\Delta t} \eta_{k}+\frac{\sigma_{2}^{2} y_{k}^{2}}{2}\left(\eta_{k}^{2}-1\right) \Delta t
\end{array}\right.
$$

where $\xi_{k}, \eta_{k}(k=1,2, \ldots)$ are two independent Gaussian random variables, and both obey the normal distribution with mean 0 and variance 1. 
Example 5.1 Choose the noise intensities $\sigma_{1}=0.2, \sigma_{2}=2$ in the stochastic tumorimmune model (1.2). Then we have

$$
\alpha-\frac{\sigma_{2}^{2}}{2}=-0.364<0 .
$$

Theorem 4.2 tell us that the density of tumor cells $y(t)$ is exponentially decreasing, see the right side of Figure 1. Meanwhile, Theorem 4.2 also shows that the distribution of effector cells $x(t)$ converges to the unique invariant probability measure $\pi_{1}(\cdot)$, the inverse gamma distribution with $a_{3}=19.715$ and $b_{3}=5.905$. To further illustrate the result of Theorem 4.2, we use the K-S test with a significance level of 0.05 to check if the stationary distribution of $x^{-1}(t)$ is the gamma distribution. At this level of significance, by Matlab we do confirm that the stationary distribution of $x^{-1}(t)$ is the Gamma distribution. Since $x^{-1}(t) \sim G(19.715,5.905)$ is equivalent to $x(t) \sim I G(19.715,5.905)$, we know that the stationary distribution of $x(t)$ is the inverse gamma distribution.
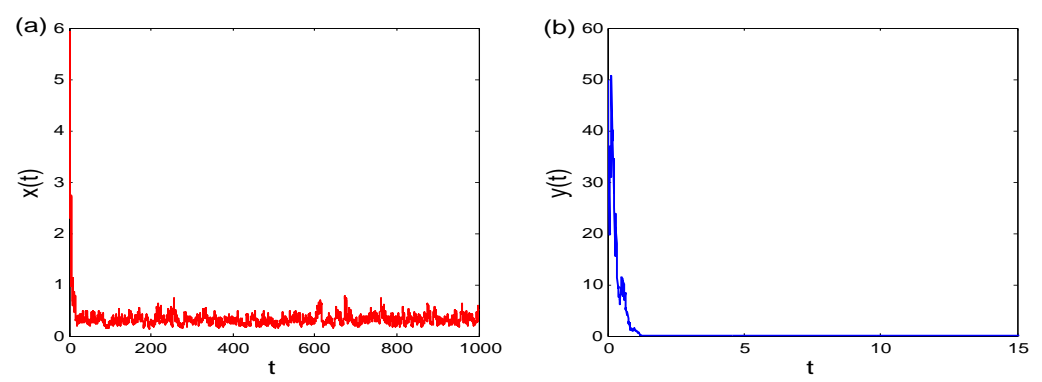

Figure 1: Example 5.1. For the stochastic tumor-immune model (1.2), the red solid line depicts the density of the effector cells $x(t)$, the solid blue line depicts the density of the tumor cells $y(t)$.

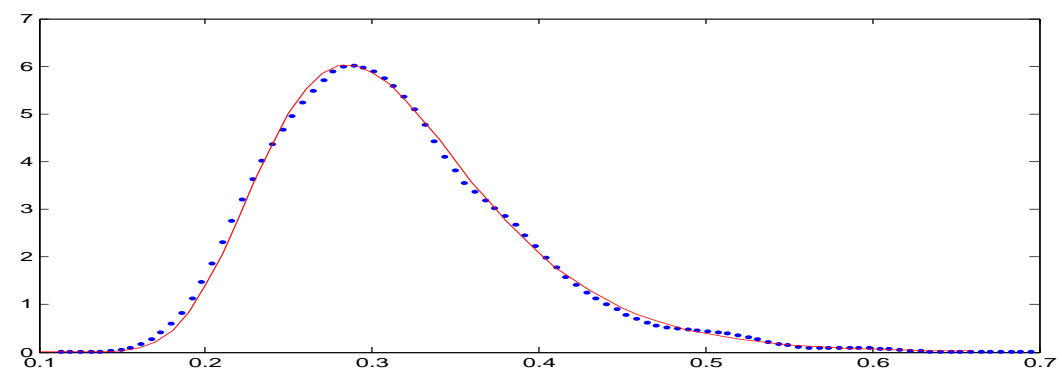

Figure 2: Example 5.1. The red solid line indicates the density function of the inverse gamma $I G(19.715,5.905)$, the blue dotted line indicates the empirical density function of the effector cell $x(t)$. 

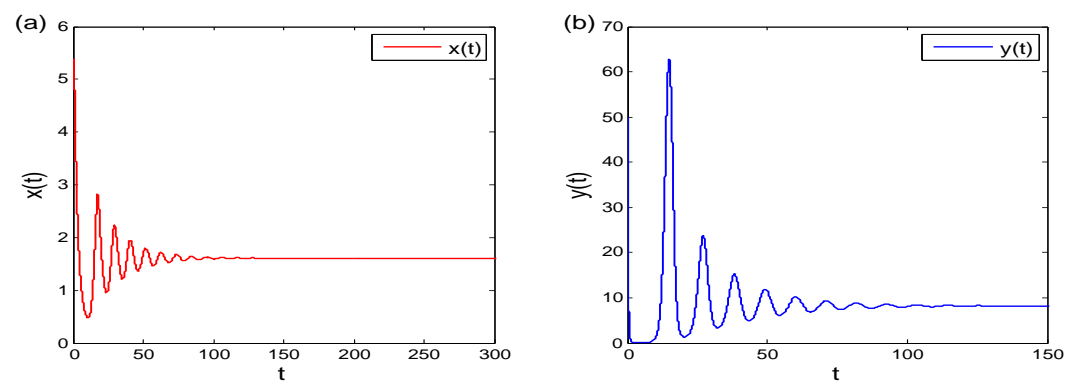

Figure 3: Example 5.1. For the deterministic tumor-immune model (1.1), the red solid line depicts the density of effector cells $x(t)$, the blue solid line depicts the density of tumor cells $y(t)$.

Furthermore, to more intuitively illustrate the result of Theorem 4.2, we plot the empirical density function of $x(t)$ and the density function of the inverse gamma distribution $I G(19.715,5.905)$ in Figure 2. One observes obviously from the Figure 2 that as $t \rightarrow \infty$, the distribution of $x(t)$ converges to $\pi_{1}(\cdot)$. Thus, this example illustrates the significance of the result of Theorem 4.2.

We go a further step to compare the dynamical behaviors of the stochastic model (1.2) with those of the deterministic model (1.1) for the same parameter values above. In [22] the authors revealed that the density of tumor cells beginning from some initial values approaches the dormant steady state while the tumor escapes the immune regulation for others initial conditions. The bifurcation analysis for parameters $\delta$ and $\sigma$ helps the illustration of the phenomenon "sneaking through". Figure 3 depicts that the path of $y(t)$ in the deterministic model tends to a positive equilibrium with frequency vibration, namely, the density of tumor cells approaches the dormant steady state. However, when the noise intensity is large satisfying $\alpha<\sigma_{2}^{2} / 2$, by virtue of Theorem 4.2,y(t) in the stochastic model (1.2) is exponentially decreasing, so the tumor cells are extinct. Figure 1 depicts the path of $y(t)$. It is revealed that stochastic perturbations for tumor cannot be ignored, and their existence plays a key role in the elimination of the tumor cells.

Example 5.2 In the stochastic tumor-immune model (1.2), let $\sigma_{2}=0.25$, which implies that the stochastic environment has a weak effect on the intrinsic growth rate of tumor cells. At the same time, the binding rate of EC to TC will be decreased when the immune response of the effector cells to the tumor cells is weak or the tumor cells are less irritating to the effector cells. Therefore, in this example we reduce the binding rate $k_{1}$ of EC and TC in the literature [22], let $\rho=0.613$. Compute

$$
\delta-h^{2}=0.09089>0, \quad \alpha-\frac{\sigma_{2}^{2}}{2}-\frac{\sigma}{\delta-h^{2}}=0.30539>0 .
$$

The empirical density of the invariant measure $\pi^{*}$ of the stochastic tumor-immune model (1.2). These imply that the conditions of Theorems 4.7 and 4.8 hold. By virtue 

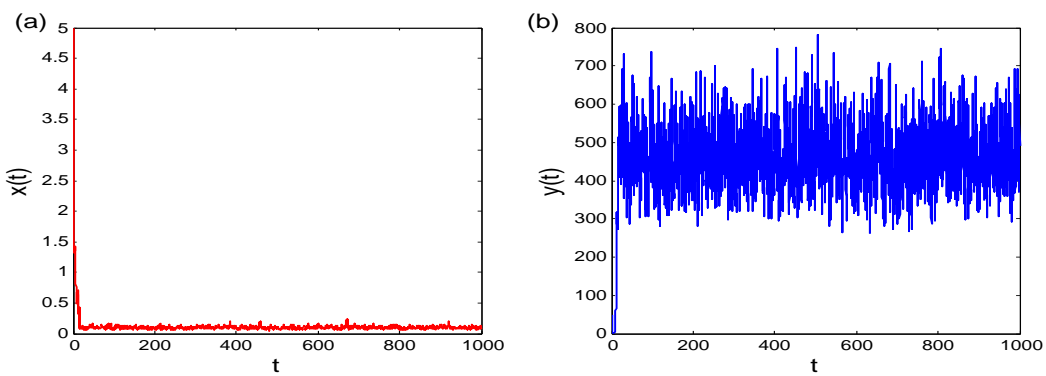

Figure 4: Example 5.2. For stochastic tumor-immune model (1.2), the red solid line indicates the density of the effector cells $x(t)$, the solid blue line indicates the density of the tumor cells $y(t)$.

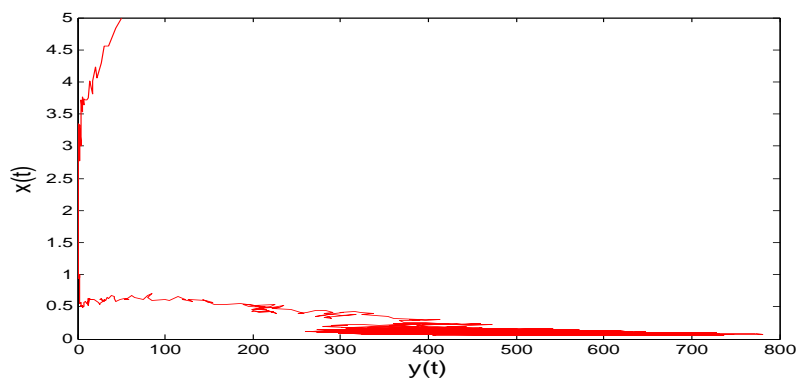

Figure 5: Example 5.2. The phase diagram of the stochastic tumor-immune model (1.2).

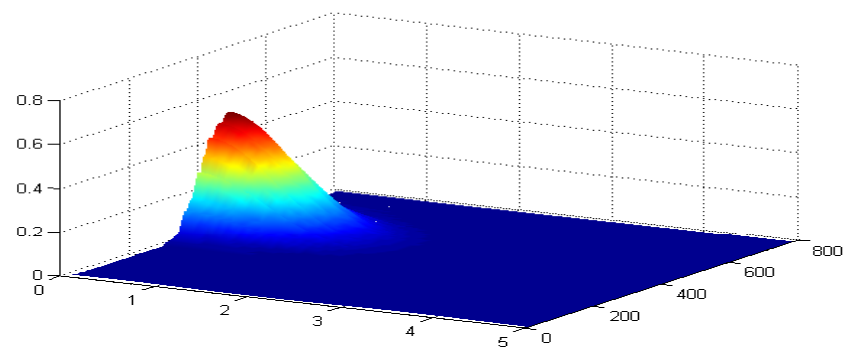

Figure 6: Example 5.2. The empirical density of the invariant measure $\pi^{*}$ of the stochastic tumor-immune model (1.2). 
of Theorems 4.7 and 4.8 the solution $(x(t), y(t))$ of the stochastic tumor-immune model (1.2) has a unique invariant probability measure $\pi_{2}(\cdot)$, and the system is stochastically permanent. Figure 4 depicts the trajectories of the effector cells $x(t)$ and the tumor cells $y(t)$ in (1.2). Figure 5 is the phase diagram with respect to the model (1.2). Figure 6 depicts the empirical density of the invariant measure $\pi_{2}(\cdot)$ of the stochastic model (1.2). Therefore, this example verifies the theoretical results of Theorems 4.7 and 4.8 well.

Under the same parameter values of this example the deterministic model in [22] predicts the existence of the dormant tumor steady state which implies the lethal tumor cells persist with little or no increase in the population. This fact is also revealed in stochastic version by the model (1.2) given that the intrinsic growth rate of tumor cells are subject to the weak stochastic perturbation.

\section{Concluding remarks}

This paper mainly studies the dynamical behaviors of the tumor-immune model proposed by [22] perturbed by the environment noise. Firstly, we prove the existence and uniqueness of the global positive solution for the tumor-immune system by the method of stochastic Lyapunov analysis. Next, by looking at the boundary and constructing appropriate comparison equations, we obtain the asymptotic moment boundedness of the effector cells and the tumor cells. Regarded the boundary equation (4.8) as a bridge, it is pointed out that when tumor cells are subject to the strong noise, the density of the tumor cells decays to zero at an exponential rate while the density of effector cells tends to a stationary distribution. Furthermore, when the noise intensity of tumor cells is small relatively, by analyzing the upper and lower limits of the density of tumor cells and effector cells at time-average, we prove the existence and uniqueness of the stationary distribution of the stochastic tumor-immune model (1.2). Moreover, the ergodicity and the stochastic permanence is obtained. Finally, all of our main results are illustrated and verified by numerical simulations. Overall, the fact is revealed that the intensity of stochastic noise for tumor cells plays a key role in the elimination of tumor cells. Compared with the deterministic tumour-immune model (1.1), the dynamical behaviors of the stochastic model under strong tumor noises are completely different. On the other hand, the phenomenon that the concentration of tumor cells tends to the dormant steady state is verified by the stochastic tumor-immune model (1.2) under weak tumor noises.

\section{Acknowledgements}

The authors would like to thank the associate editor and referees for the helpful comments and suggestions. 


\section{References}

[1] J.A. Adam, N. Bellomo. A Survey of Models for Tumor-Immune System Dynamics. Birkhäuser, Boston, 1997.

[2] G. Albano, V. Giorno. A stochastic model in tumor growth. Journal of Theoretical Biology, 2006, 242(2): 329-366.

[3] R.P. Araujo, D.L.S. McElwain. A history of the study of solid tumour growth: the contribution of mathematical modelling. Bulletin of Mathematical Biology, 2004, 66(5): 1039-1091.

[4] V.I. Bogachev, N.V. Krylov, M. Röckner. On regularity of transition probabilities and invariant measures of singular diffusions under minimal conditions. Comm. Partial Differential Equations, 2001, 26(11-12): 2037-2080.

[5] J. Bao, J. Shao. Permanence and extinction of regime-switching predator-prey models. SIAM Journal on Mathematics and Applications, 2016, 48(1): 725-739.

[6] N. Bellomo. Modeling Complex Living Systems: A Kinetic Theory and Stochastic Game Approach. Birkhäuser, Bosten·Basel·Berlin, 2007.

[7] R. Bürger, The Mathematical Theory of Selection, Recombination, and Mutation. John Wiley \& Sons, Ltd., Chichester, 2000.

[8] L.G. de Pillis, A.E. Radunskaya, C.L. Wiseman. A validated mathematical model of cell-mediated immune response to tumor growth. Cancer Research, 2005, 65(17): 7950-7958.

[9] N.T. Dieu, D.H. Nguyen, N.H. Du, G. Yin. Classification of asymptotic behavior in a stochastic SIR model. SIAM Journal on Applied Dynamical Systems, 2016, 15(2): 1062-1084.

[10] A. d'Onofrio. A general framework for modeling tumor-immune system competition and immunotherapy: Mathematical analysis and biomedical inferences. Physica D. Nonlinear Phenomena, 2005, 208(3-4): 220-235.

[11] N.H. Du, D.H. Nguyen, G. Yin. Conditions for permanence and ergodicity of certain stochastic predator-prey models. Journal of Applied Probability, 2016, 53(1): 187202.

[12] R.L. Elliott, G.C. Blobe. Role of transforming growth factor Beta in human cancer. Journal of Clinical Oncology Official Journal of the American Society of Clinical Oncology, 2005, 23(9): 2078-2093.

[13] L. Ferrante, S. Bompadre, L. Possati, L. Leone. Parameter estimation in a Gompertzian stochastic model for tumor growth. Biometrics, 2000, 56(4): 1076-1081. 
[14] R.P. Garay, R. Lefever. A kinetic approach to the immunology of cancer: stationary states properties of effector-target cell reactions. Journal of Theoretical Biology, 1978, 73(3): 417-438.

[15] N.S. Goel, N. Richter-Dyn. Stochastic Models in Biology. Academic Press, New York, 1975.

[16] A. Hening, D.H. Nguyen, Coexistence and extinction for stochastic Kolmogorov systems. Ann. Appl. Probab. 2018, 28 (3): 1893-1942.

[17] A. Hening, D.H. Nguyen, G. Yin, Stochastic population growth in spatially heterogeneous environments: the density-dependent case. J. Math. Biol. 2018, 76(3): 697-754.

[18] D.J. Higham. An algorithmic introduction to numerical simulation of stochastic differential equations. SIAM Review, 2001, 43(3): 525-546.

[19] K. Ichihara, H. Kunita. A classification of the second order degenerate elliptic operators and its probabilistic characterization. Zeitschrift für Wahrscheinlichkeitstheorie und Verwandte Gebiete, 1974, 30: 235-254.

[20] R. Khasminskii. Stochastic Stability of Differential Equations, 2nd edn. Springer, Heidelberg, 2012.

[21] N.L. Komarova, D. Wodarz, E. Angelis, M.A.J. Chaplain, N. Bellomo. Selected Topics in Cancer Modeling: Genesis, Evolution, Immune Competition, and Therapy. Birkhäuser, Boston·Basel-Berlin, 2008.

[22] V.A. Kuznetsov, I.A. Makalkin, M.A. Taylor, A.S. Perelson. Nonlinear dynamics of immunogenic tumors: Parameter estimation and global bifurcation analysis. Bulletin of Mathematical Biology, 1994, 56(2): 295-321.

[23] D. Li, F. Cheng. Threshold for extinction and survival in stochastic tumor immune system. Communications in Nonlinear Science and Numerical Simulations, 2017, 51: $1-12$.

[24] K.J. Mahasa, R. Ouifki, A. Eladdadi, L.G. de Pillis. Mathematical model of tumorimmune surveillance. Journal of Theoretical Biology, 2016, 404: 312-330.

[25] A. Mantovani, P. Allavena, A. Sica. Tumour-associated macrophages as a prototypic type II polarised phagocyte population: role in tumour progression. European Journal of Cancer, 2004, 40(11): 1660-1667.

[26] X. Mao, C. Yuan. Stochastic Differential Equations with Markovian Switching. Imperial College Press, London, 2006. 
[27] S.P. Meyn, R.L. Tweedie. Stability of Markovian processes III: Foster-Lyapunov criteria for continuous-time processes. Advances in Applied Probability, 1993, 25(3): 518-548.

[28] B. Mukhopadhyay, R. Bhattacharyya. A nonlinear mathematical model of virustumor-immune system interaction: deterministic and stochastic analysis. Stochastic Analysis and Applications, 2009, 27(2): 409-429.

[29] M.A. Nowak, R.M. May. Virus Dynamics: Mathematical Principles of Immunology and Virology. Oxford University Press, Oxford, 2000.

[30] C. Oana, O. Dumitru, C. Riccardo. Review of stochastic stability and analysis tumorimmune systems. Current Bioinformatics, 2013, 8(4): 390-440.

[31] M. Owen, J. Sherratt. Modelling the macrophage invasion of tumors: Effects on growth and composition. IMA Journal of Mathematics Applied in Medicine \& Biology, 1998, 15(2): 165-185.

[32] A.S. Perelson, G.Weisbuch. Immunology for physicists. Review of Modern Physics, 1997, 69(4): 1219-1267.

[33] H. Siu, E.S. Vitetta, R.D. May, J.W. Uhr. Tumor dormancy. I. Regression of BCL1 tumor and induction of a dormant tumor state in mice chimeric at the major histocompatibility complex. Journal of Immunology, 1986, 137(4): 1376-1382.

[34] W.Y. Tan, C.W. Chen. "Cancer stochastic models," in Encyclopedia of Statistical Sciences, John Wiley \& Sons, Ltd., New York, 2006.

[35] M. Villasana, A. Radunskaya. A delay differential equation model for tumor growth. Journal of Mathematical Biology, 2003, 47(3): 270-294.

[36] J.T. Wu, D.H. Kirn, L.M. Wein. Analysis of a three-way race between tumor growth, a replication-competent virus and an immune response. Bulletin of Mathematical Biology, 2004, 66(4): 605-625.

[37] R. Yafia. Hopf bifurcation in differential equations with delay for tumor-immune system competition model. SIAM Journal on Applied Mathematics, 2007, 67(6):16931703. 